\title{
Cerenkov emission as a positive tag of double beta decays in bolometric experiments
}

\author{
T. Tabarelli de Fatis ${ }^{\mathrm{a}}$ \\ Dipartimento di Fisica, Università di Milano Bicocca, Piazza della Scienza 3, 20133 Milano, Italy
}

Received: 17 August 2009 / Revised: 26 October 2009 / Published online: 19 November 2009

(C) Springer-Verlag / Società Italiana di Fisica 2009

\begin{abstract}
Active background reduction in high resolution calorimeters is a promising approach to achieve ultimate sensitivity in neutrinoless double beta decay experiments. We propose Cerenkov emission from beta rays in bolometric crystals as a viable alternative to scintillation. This novel approach could broaden the range of materials of interest for calorimetric searches of the double beta decay. We discuss the optical properties of $\mathrm{TeO}_{2}$ crystals, as a show case.
\end{abstract}

PACS 23.40.-s · 07.57.Kp · 41.60.Bq

\section{Introduction}

Neutrinoless double beta decay is a sensitive probe of the charge conjugation properties of neutrinos and of the neutrino mass scale. This decay is expected to occur for several even-even nuclei at a (tiny) rate proportional to the square of the effective Majorana mass of the electron neutrino. Neutrino oscillations results suggest that the effective electron neutrino mass be of order $20-50 \mathrm{meV}$ or below $10 \mathrm{meV}$ for inverted or direct hierarchy respectively, unless the CP-phases of Majorana neutrinos conspire for cancellations. For this low mass scales to be tested, sensitivities to half-life time of the candidate nuclei of order $10^{26}-10^{27} \mathrm{y}$ need to be achieved.

The experimental signature for the decay is rather clean in itself and corresponds to a peak, only smeared by the instrumental resolution, in the spectrum of the energy sum of the two electrons emitted in the decay. Yet, the search for such a rare decay is thwarted by a background of spurious events due to radioactive contaminants in the environment and in the detector material.

The struggle for background reduction in double beta decay experiments is documented by decades of research

a e-mail: tommaso.tabarelli@cern.ch and development projects in several directions, exploiting a variety of experimental techniques. In addition to careful selections of materials with low radioactive contaminants, merit factors in modern experiments are the detector resolution and dual readout techniques to provide further rejection power. A detailed comparison of the different techniques is beyond the scope of this paper and the reader is referred to review articles on the subject [1]. Here we propose that a dual readout approach based on the detection of Cerenkov photons emitted by beta rays could win a sizable background rejection in bolometric experiments. This option, supplementary or even alternative to scintillation, may open the window to the search for new non-scintillating materials for double beta decay searches. In addition, it may already offer an interesting readout option for current experiments. As show case, we discuss a potential application to the CUORE experiment [2], an array of high resolution bolometric calorimeters based on $\mathrm{TeO}_{2}$ crystals.

\section{Dual readout approaches in bolometric experiments}

Bolometers are crystals operated at cryogenic temperatures, where the low heat capacity implies a sizable (measurable) variation of the crystal temperature upon the energy deposit of a particle. This technique has long been pioneered with $\mathrm{TeO}_{2}$ crystals and has now grown into one of the leading experiments in the field. Yet, the experiment is plagued by an important background due to alpha decays with energy deposits non fully contained in a crystal, due to residual contaminants in the crystal itself, on its surfaces or in the materials of the mechanical supports. Alpha particles depositing only a fraction of their energy in the bolometer volume give rise to a continuum in the energy spectrum, which reduces the sensitivity to the feeble double beta decay signal, expected at around $2.53 \mathrm{MeV}$ for ${ }^{130} \mathrm{Te}$. A detailed analysis of the spurious events recorded by the CUORE ancestor, the Cuoricino setup [3], demonstrated that about $2 / 3$ of the background at 
the energy of the expected neutrinoless double beta decay transition can be ascribed to energy-degarded alpha events and $1 / 3$ to gamma rays from the radioactive contaminants in the cryostat materials. In CUORE, the background is expected to be dominated by alpha events, while the gamma contribution to the background will be reduced to a negligible level, through an improved design of passive shields inside the cryostat [2].

Several approaches for active background reduction against alpha events have been proposed, based on the anticoincidence of nearby crystals or on the use of designated veto $\mathrm{Si}$ bolometers mounted on the $\mathrm{TeO}_{2}$ surfaces [4, 5]. Alternatively, a dual readout approach based on the simultaneous measurement of the bolometric signals and of the scintillation light has been advocated, with applications both in double beta decay and in Dark Matter searches [6-10]. The rationale of this technique is that the scintillation light due to heavy particles is strongly quenched and the measurement of the ratio of the emitted light over the energy dissipated in heat allows one for signal-background discrimination. While recent results in this direction are encouraging on some crystals $[11,12]$, this approach is only limited to materials that exhibit some scintillation property. The exploitation of this techniques with $\mathrm{TeO}_{2}$ is frustrated by the low luminescence of this crystal, which is also critically dependent on the quality of the crystal growth and on the choice of suitable dopants. In spite of these difficulties, the interest of the potential application has prompted for some dedicated R\&D efforts [13-16]. As an alternative to these efforts, we suggest that standard $\mathrm{TeO}_{2}$ crystals have suitable optical properties to act as excellent Cerenkov radiator. At variance with scintillation properties, the optical parameters are less dependent on crystal growth conditions. As we show in the next section, the Cerenkov light emitted by fast electrons can be exploited to efficiently tag double beta decay events against the alpha background.

\section{The Cerenkov yield \\ from neutrinoless double beta decay of ${ }^{130} \mathrm{Te}$ in $\mathrm{TeO}_{2}$ crystals}

$\mathrm{TeO}_{2}$ is a clear crystal, transparent to light from about $350 \mathrm{~nm}$ to the infrared region, and it has index of refraction of about $n=2.4$ [17], corresponding to a threshold for Cerenkov emission of about $50 \mathrm{keV}$ for electrons and about $400 \mathrm{MeV}$ for alpha particles. Thus, at variance with scintillation, no Cerenkov emission is expected form the alpha background and a positive tag of a double beta decay with full background rejection could be achieved, ideally, even upon the detection of one single Cerenkov photon.

This consideration, though still qualitative, open new alleys to dual readout approaches in bolometric experiments, making compounds with null or weak scintillation potentially as attractive as scintillators. In case of pure Cerenkov radiators the signature would be clean; in case of weak scintillators, such as $\mathrm{TeO}_{2}$, time and spectral properties of the Cerenkov emission can be exploited to select Cerenkov photons at readout. Less aggressively, Cerenkov emission can be considered as supplementary to scintillation to improve signal to background discrimination.

A quantitative estimate of the Cerenkov yield from the two electrons emitted in the neutrinoless double beta decay of ${ }^{130} \mathrm{Te}$ has been derived from the above-mentioned optical properties of the crystal and from electron range tables for a $6 \mathrm{~g} / \mathrm{cm}^{3}$ density $\mathrm{TeO}_{2}$ crystal [19]. The energy spectrum of the two electrons in the pair has been simulated according to the parametrisation $d N / d T_{e} \propto\left(T_{e}+1\right)^{2}\left(Q+1-T_{e}\right)^{2}$, where $T_{e}$ is the kinetic energy of the electron and $Q$ is the transition energy [20]. From the above, we calculate that the two electrons emit in total along their range about 125 Cerenkov photons in an energy interval from $2 \mathrm{eV}$ (about $600 \mathrm{~nm}$ ) to $3.5 \mathrm{eV}$ (about $350 \mathrm{~nm}$ ). This emission corresponds to an energy efficiency of about $140 \mathrm{eV} / \mathrm{MeV}$, about two order of magnitude larger than the observed emission from alpha particles in $\mathrm{TeO}_{2}$ crystals [13], though the latter results are not corrected for detection efficiency. Fluctuations around the above value are expected from Poisson statistics and from the sharing of the energy between the two electrons. As we show in Fig. 1, the Cerenkov yields from the sum of the two electrons is practically independent of the energy sharing between the two electrons. The relative yield increases by less than about $10 \%$, when one of the electrons takes the entire decay energy. This figure is comparable or

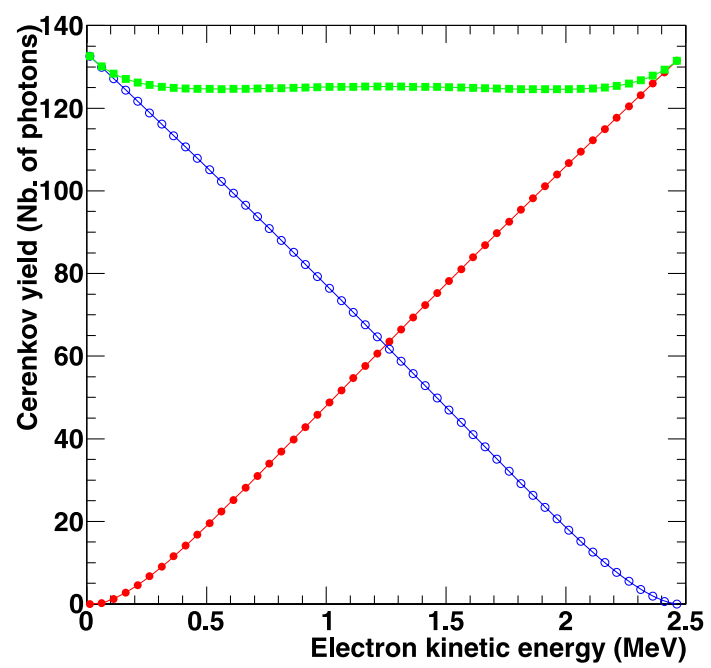

Fig. 1 Calculated Cerenkov yield from $350 \mathrm{~nm}$ to $600 \mathrm{~nm}$ in $\mathrm{TeO}_{2}$ as a function of the kinetic energy of one of the two electrons emitted in the $\beta \beta$-decay of ${ }^{130} \mathrm{Te}$. The three curves show the yield associated to that electron (full dots), the yield of the other electron (open dots) and the total yield from the sum of the two electrons (squares) 
lower than the expected stochastic fluctuations associated to light emission and detection.

The optical properties of $\mathrm{TeO}_{2}$ crystals are slightly temperature dependent. In particular, the absorption edge drifts towards lower wavelengths at low temperature, implying an increased transparency down to $300 \mathrm{~nm}$ [14-16]. The index of refraction decreases with temperature, with a variation of less than 5\% for over a range of $800 \mathrm{~K}$ [18]. Direct measurements below $77 \mathrm{~K}$, where $n$ is about $0.6 \%$ lower than at room temperature are outstanding. Moreover, the index of refraction has a $5-10 \%$ variation depending on the polarization state of the light. These figures do not substantially modify our prediction and have no impact on the global perspective of the paper.

On the basis of the curves shown in Fig. 1, one might argue that Cerenkov emission could also provide some discrimination power against gamma-induced background events. In this case, at variance with beta rays, the energy is deposited through multiple interactions, in which the photon energy is transferred to low energy electrons. For a multiplicity $N$, a Cerenkov yield equivalent to the one from a single electron of kinetic energy equal to $E_{\gamma}-(N-1) \times E_{\mathrm{th}}$, where $E_{\gamma}$ and $E_{\text {th }}$ are the photon energy and the effective energy threshold for Cerenkov emission, is expected. We have not addressed this in a detailed study, but simple arguments based on Fig. 1 indicate that any potential discrimination power is spoiled by stochastic fluctuations, when light collection and quantum efficiencies are considered.

Light collection and quantum efficiencies may constitute a serious experimental challenge at cryogenic temperatures [6-12]. Yet, as discussed above, Cerenkov light offer the unique opportunity of full alpha background rejection even in the limit of very low detection efficiency. While conventional Si photon-detectors can easily reach single photoelectron sensitivities at room temperature, early attempts to operate these devices at cryogenic temperatures were abandoned, because of technical difficulties [6, 7]. Alternatively, light absorbers operated as bolometers can be used. The optimization of such devices for Dark Matter searches has already reached sensitivities to light yields comparable or lower to the ones predicted in this paper for the Cerenkov emission associated to double beta decays [8-10]. In conclusion, although a dedicated R\&D work is needed for the specific application discussed in this paper, existing experimental techniques seem to offer already the sensitivity required for the detection of the low light levels considered.

\section{Summary}

We propose that the use of Cerenkov radiation emitted by the two electrons from neutrinoless beta decay can be used for active background reduction purposes in double beta decay experiments. The peculiarity of the Cerenkov emission, totally absent for alpha particles in the energy range relevant to double beta decays, qualify this process as alternative to active background reduction approaches based on the scintillation properties of materials. This could broaden the set of interesting materials for double beta decay experiments. Particularly interesting would be the search for new compounds with double beta candidates with a transition energy above $2.614 \mathrm{MeV}$ : the energy of the highest gamma ray from natural radioactivity.

Restricting the discussion to known materials, we have analysed the properties of $\mathrm{TeO}_{2}$ crystals, as a show case. This compound, known as weak scintillator, is adopted by the CUORE observatory, one of the most sensitive planned experiments, though plagued by a substantial background of alpha induced spurious events. We estimate a total yield of about 125 Cerenkov photons per neutrinoless double beta decay of ${ }^{130} \mathrm{Te}$, prior to quantum efficiency and light collection effects. This light emission is detectable by available experimental techniques. In particular, low light level detectors developed for Dark Matter searches seem to match the required performance. This could permit the rejection of the alpha induced spurious events, expected to be the dominant contribution to the background in the region where the double beta decay signal is expected.

Acknowledgements Discussions with O. Cremnonesi, S. Ragazzi and A. Vedda and stimulating comments from the referees are acknowledged.

\section{References}

1. F.T. Avignone et al., Rev. Mod. Phys. 80, 481 (2008)

2. R. Ardito et al. (The CUORE Collaboration), Prog. Part. Nucl. Phys. 57, 203 (2006)

3. C. Arnaboldi et al., Phys. Rev. C 78, 035502 (2008)

4. L. Foggetta et al., Appl. Phys. Lett. 86, 134106 (2005)

5. M. Pedretti et al., AIP Conf. Proc. 897, 59-64 (2007)

6. A. Alessandrello et al., Nucl. Phys. B (proc. Suppl.) A 28, 233 (1992)

7. A. Alessandrello et al., IEEE Trans. Nucl. Sci. 39(4), 612 (1992)

8. C. Bobin et al., Nucl. Instrum. Methods A 386, 453 (1994)

9. F. Petricca et al., Nucl. Instrum. Methods A 520, 193 (2004)

10. G. Angloher et al., Astropart. Phys. 23, 325 (2005)

11. S. Pirro et al., Phys. At. Nucl. 69, 2109 (2006)

12. L. Gironi et al., arXiv:0809.5126 [nucl-ex]

13. N. Coron et al., Nucl. Instrum. Methods A 520, 159 (2004)

14. I. Dafinei et al., Nucl. Instrum. Methods A 554, 195 (2005)

15. I. Dafinei et al., Phys. Status Solidi (a) 204(5), 1567 (2007)

16. I. Dafinei et al., in Scint2005 Proc. 8th Int. Conf. on Inorganic Scintillators and their Use in Scientific and Industrial Applications, Alushta, Crimea, Ukraine, 19-23 September 2005, pp. 106108

17. Handbook of Optics, vol. 2, 2nd edn. (McGraw-Hill, 1994)

18. I.V. Stefanskii et al., J. Appl. Spect. 51(2), 790 (1989)

19. Stopping-power and range tables for electrons, ICRU Report No. 37, 1984

20. H. Primakoff, S. Rosen, Rep. Prog. Phys. 22, 121-166 (1959) 